\title{
The enigma that is the nucleus pulposus cell: the search goes on
}

\author{
W Mark Erwin \\ See related research by Gilson et al., http://arthritis-research.com/content/12/1/R24
}

\begin{abstract}
The development of an effective treatment for degenerative disc disease has been hampered for many years by what seems a fundamental problem; what exactly defines a nucleus pulposus (NP) cell? The paper by Gilson and colleagues elegantly re-opens the debate concerning the lineage and identity of NP cells that are alike yet different from chondrocytes. As we pursue novel investigations and treatment strategies for degenerative disc disease, how do we isolate these unique cells and what is the role of the primordial notochordal cell that may well linger within the NP far longer and perhaps in a different phenotypic appearance than previously thought? The paper by Gilson and colleagues that is the subject of the present editorial presents compelling data concerning the heterogeneity of the cells of the NP, and their origin, development, maturation and function.
\end{abstract}

A recent issue of Arthritis Research and Therapy contains a report by Gilson and colleagues describing their investigation of the differential cell surface marker expression found in samples of bovine intervertebral disc (IVD) and articular chondrocytes [1]. This report raises interesting questions about the identity of the residents within the nucleus pulposus (NP) and has broad implications with respect to regenerative medicine and tissue engineering of the IVD.

A recent search of PubMed using the search term 'tissue engineering and intervertebral disc' returned 263 hits, with the oldest publication dating to 1989. Although investigators have clearly been interested in developing a biological treatment for degenerative disc disease for

*Correspondence: mark.erwin@utoronto.ca

The Spine Programme, Division of Orthopaedic Surgery, Toronto Western Hospital, University of Toronto, 399 Bathurst Street, Rm 13-310 McLaughlin Pavilion, Toronto, Ontario, Canada, M5T 258 over 30 years, we must be in the early days since we have yet to characterize the ubiquitous NP cell or to really understand the composition of the NP cellular milieu.

The flexible model of cell and tissue classification whereby expression patterns reflect a functional approach rather than strict germ layer derivation suggests that, with respect to the identity of NP cells, there may be more than meets the eye [2].

The notochord derives from all three germ layers as it originates in a blended fashion from primitive ectoderm, sharing mesodermal and endodermal attributes as it develops as an outgrowth from Hensen's node between the ectoderm and endoderm [3]. This co-joined origin is particularly poignant in human and other mammals, as distinct from lower animals, because in higher mammals the developing notochord provides a pathway for migration of ectoderm to endoderm [3]. The presence of vimentin in NP cells suggests that motility may play a role in the development of the NP cellular composition; perhaps including cells that migrate inwards from the vertebral endplates [4]. As Gilson and colleagues have reported, however, the cells occupying the NP may change their appearance over time, masking their original phenotype but perhaps retaining some of their original capacity. Have these cells altered their phenotype as a consequence of maturation or pathological events, or as an adaptive response to life in the disc over time?

The IVD is a hypoxic, isolated, immune-privileged compartment, the cells of which must necessarily be highly specialized in order to survive. Classically it has been thought that once the NP has been formed the notochordal cells disappear, leaving behind the fibrocartilagenous NP cell. But then along come Gilson and colleagues - who find that within adult bovine caudal discs (a tissue compartment formerly thought to be fairly homogeneous) there exists a small percentage of notochordal holdouts that continue to express their notochordal lineage markers. Is it that a small, primordial notochordal cell reservoir may linger longer than was previously thought within the mature NP?

These observations raise a number of questions notably, is the protection from degenerative disc disease 
seen in species that retain their notochordal cell-rich appearance, such as the nonchondrodystrophic canine and rabbit, due to the differential extracellular matrix synthesized by these cells as compared with the NP cell? [5]. Is it a dose-response issue whereby the discs that are relatively deficient in notochordal cells are therefore lacking in the necessary and sufficient molecules synthesized by these cells that may act upon the NP cell $[6,7]$ ? It is thought that the notochordal cell-rich disc NP phenotype confers superior biomechanical properties $[5,8]$. Do notochordal cell-deficient discs therefore fail to resist the loads imposed by daily life over time due to biomechanical or biochemical reasons - or both? Also, and importantly from the perspective of evaluating putative therapies, which cells are the best to use for in vitro assays? Should future NP cell experiments exclude cytokeratin- $8^{+}$cells or does this matter when evaluating the mechanisms of the IVD NP as an organ?

In terms of the progression of degenerative disc disease, the NP could arguably represent the lynchpin in the degenerative cascade since many investigators consider the NP as the area demonstrating the earliest degenerative changes [9-11]. We may therefore need to look ever closer at the question of what really defines the cells within the disc. Are the current models of events leading to failure of the disc as an organ correct? What role(s) do the cells play within the NP that may mitigate or contribute to the progression of organ failure?

As we look to the future and contemplate cell-based therapeutics for the treatment of degenerative disc disease, one must wonder what might be the most appropriate source of cells. Bone marrow-derived stem cells originate within an entirely different niche to cells that have adapted to survive within the NP with its tenuous nutrient supply and hypoxic environment. Along which lineage should stem cells or progenitor cells therefore be directed in order to potentially repopulate the disc and how would they best be able to restore homeostasis? For now, given that the mature disc nucleus contains holdouts of the primitive notochordal cell, perhaps the best perspective from which to answer these questions is one where we take a fresh look back at the origin, development and maturation of the IVD.

\section{Abbreviations}

IVD, intervertebral disc; NP, nucleus pulposus.

\section{Competing interests}

The author declares that he has no competing interests.

Published: 24 May 2010

\section{References}

1. Gilson A, Dreger M, Urban JP: Differential expression level of cytokeratin 8 in cells of the bovine nucleus pulposus complicates the search for specific intervertebral disc cell markers. Arthritis Res Ther 2010, 12:R24.

2. Viebahn C, Lane EB, Ramaekers FC: Keratin and vimentin expression in early organogesis of the rabbit embryo. Cell Tissue Res 1988, 253:553-562.

3. Gajović S, Kostović-Knezević L, Svajger A: Origin of the notochord in the rat embryo tail. Anat Embryol 1989, 179:305-310.

4. Kim K-W, Lim T-H, Kim JG, Jeong S-T, Masuda K, An HS: The origin of chondrocytes in the nucleus pulposus and histologic findings associated with the transition of a notochordal nucleus pulposus to a fibrocartilaginous nucleus pulposus in intact rabbit intervertebral discs. Spine 2003, 28:982-990

5. Cappello R, Bird JLE, Pfeiffer D, Bayliss MT, Dudhia J: Notochordal cell produce and assemble extracellular matrix in a distinct manner, which may be responsible for the maintenance of healthy nucleus pulposus. Spine 2006, 31:873-882.

6. Erwin WM, Inman RD: Notochord cells regulate intervertebral disc chondrocyte proteoglycan production and cell proliferation. Spine 2006, 31:1094-1099.

7. Erwin WM, Ashman K, O'Donnel P, Inman RD: Nucleus pulposus notochord cells secrete connective tissue growth factor and upregulate proteoglycan expression by intervertebral disc chondrocytes. Arthritis Rheum 2006, 54:3859-3867.

8. Oegema JrTR: The role of disc cell heterogeneity in determining disc biochemistry: a speculation. Biochem Soc Trans 2002, 30:839-844.

9. Haefeli M, Kalberer F, Saegesser D, Nerlich AG, Boos N, Paesold G: The course of macroscopic degeneration in the human lumbar intervertebral disc. Spine 2006, 31:1522-1531.

10. Adams MA, Roughley PJ: What is intervertebral disc degeneration and what causes it? Spine 2006, 31:2151-2161.

11. Zhao CQ, Wang LM, Jiang LS, Dai LY: The cell biology of intervertebral disc aging and degeneration. Ageing Res Rev 2007, 6:247-261.

doi:10.1186/ar3001

Cite this article as: Erwin WM: The enigma that is the nucleus pulposus cell: the search goes on. Arthritis Research \& Therapy 2010, 12:118. 